\title{
Evolutionary Biclustering with Correlation for Gene Interaction Networks
}

\author{
Ranajit Das $^{1}$, Sushmita Mitra ${ }^{1}$, Haider Banka ${ }^{2}$, and Subhasis Mukhopadhyay ${ }^{3}$ \\ ${ }^{1}$ Machine Intelligence Unit, Indian Statistical Institute, Kolkata 700 108, India \\ \{ranajit_r,sushmita\}@isical.ac.in \\ ${ }^{2}$ Center for Soft Computing Research, Indian Statistical Institute, \\ Kolkata 700 108, India \\ hbanka_r@isical.ac.in \\ ${ }^{3}$ Bioinformatics Center, Department of Bio-Physics, Molecular Biology and Genetics, \\ Calcutta University, Kolkata 700 009, India \\ smbmbg@caluniv.ac.in
}

\begin{abstract}
In this study, a novel rank correlation-based multiobjective evolutionary biclustering method is proposed to extract simple gene interaction networks from microarray data. Preprocessing helps to preserve those gene interaction pairs which are strongly correlated. Experimental results on time series gene expression data from Yeast are biologically validated based on standard databases and information from literature.
\end{abstract}

Keywords: Bioinformatics, transcriptional regulatory network extraction, gene expression profile, gene interaction network.

\section{Introduction}

Advent of DNA microarray technology, leading to complete-genome expression profiling, coupled with various analytical methods provide a lot of information about cellular function. This forms an indispensable tool for exploring transcriptional regulatory networks from the system level and is useful when one dwells into the cellular environment to investigate various complex interactions [1]. Biological pathways can be represented as networks and broadly classified as metabolic pathways, signal transduction pathways and gene regulatory networks or gene interaction networks. Biological networks connect genes, gene products (in the form of protein complexes) to one another. A network of co-regulated genes may form gene clusters that can encode proteins, which interact amongst themselves and take part in common biological processes. Clustering of gene expression profiles have been employed to identify co-expressed groups of genes [2] as well as to extract gene interaction/gene regulatory networks [3.

Sharing of the regulatory mechanism amongst genes, in an organism, is predominantly responsible for their co-expression. Genes with similar expression profiles are very likely to be regulators of one another or be regulated by some other common parent gene 4. Often, it is noted that during few conditions a small set of genes are co-regulated and co-expressed, their behavior being almost 
independent for rest of the conditions. The genes share local rather than global similar patterns in their gene expression profiles. Generally, group of genes are identified in the form of biclusters using continuous columns biclustering because biological processes start and terminate over a continuous interval of time [5], 6]. The aim of biclustering is to bring out such local structure inherent in the gene expression data matrix. It refers to the clustering of both rows (genes) and columns (conditions) of a data matrix (gene expression matrix), simultaneously, during knowledge discovery about local patterns from microarray data 7 .

In this paper we use continuous column multiobjective evolutionary biclustering to propose the extraction of rank correlated gene pairs for generating gene interaction subnetworks based on regulatory information among genes. Preprocessing, involving the discretization of the rank correlation matrix (using quantile partitioning) and subsequent elimination of weak correlation links, is employed to retain strongly rank correlated (positive or negative) gene interaction pairs. An adjacency matrix is formed from the resulting correlation matrix, based on which the network is generated and biologically validated. The usefulness of the model is demonstrated, using time-series gene expression data from Yeast.

\section{Extraction of Gene Interaction Network}

Biological networks involving gene pairs which demonstrate transcription factor $(T F)$-target relationship, is an important research problem. A gene interaction network is a complex structure comprising various gene products activating or repressing other gene products. A gene that regulates other genes is termed the transcription factor, while the gene being regulated is called its target. The presence of a TF, can alternatively switch "ON" some genes in the network while others remain "OFF", orchestrating many genes simultaneously. The proper understanding of gene interaction networks is essential for the understanding of fundamental cellular processes involving growth and decay, development, secretion of hormones, etc. During transcription of gene expression specific groups of genes may be made active by certain signals which on activation, may regulate similar biological processes. The genes may also be regulators of each others transcription. Target genes sharing common TFs demonstrate similar gene expression patterns along time [8], [9]. Analysis of similar expression profiles brings out several complex relationships between co-regulated gene pairs, including coexpression, time shifted, and inverted relationships [10.

In this paper an attempt has been made to model the relationship between a transcription factor and its target's expression level variation over time in the framework of the generated biclusters. The extraction of the relationship between the gene pair is biologically more meaningful and computationally less expensive as a bicluster is a subset of highly correlated genes and conditions. Rank correlation provides a similarity measure, which retains the relevant information necessary for computing pairwise correlation between gene pairs. The relationship is presented in terms of rules, where a $T F$ is connected to its regulated target 
gene. These rules are subsequently mapped to generate parts of the entire regulatory network. It may be noted that intra-pathway gene interactions, responsible for a particular biological function and possibly within a bicluster, are generally stronger than any inter-pathway interactions.

\subsection{Multi-objective Evolutionary Biclustering}

Biclustering refers to the simultaneous clustering and redundant feature reduction involving both attributes and samples. This results in the extraction of biologically more meaningful, less sparse partitions from high-dimensional data, and exhibit similar characteristics. The partitions are known as biclusters. Biclustering has been applied to gene expressions from cancerous tissues [11, mainly for identifying co-regulated genes, gene functional annotation, and sample classification.

A bicluster can be defined as a pair $(g, c)$, where $g \subseteq\{1, \ldots, m\}$ represents a subset of genes and $c \subseteq\{1, \ldots, n\}$ represents a subset of conditions (or time points). The optimization task 7] involves finding the maximum-sized bicluster not exceeding a certain homogeneity constraint mentioned below. The size (or volume) $f(g, c)$ of a bicluster is defined as the number of cells in the gene expression matrix $E$ (with values $e_{i j}$ ) that are covered by it. The homogeneity $\mathcal{G}(g, c)$ is expressed as a mean squared residue score. We maximize

$$
f(g, c)=|g| \times|c|,
$$

subject to a low $\mathcal{G}(g, c) \leq \delta$ for $(g, c) \in X$, with $X=2^{\{1, \ldots, m\}} \times 2^{\{1, \ldots, n\}}$ being the set of all biclusters, where

$$
\mathcal{G}(g, c)=\frac{1}{|g| \times|c|} \sum_{i \in g, j \in c}\left(e_{i j}-e_{i c}-e_{g j}+e_{g c}\right)^{2} .
$$

Here $e_{i c}=\frac{1}{|c|} \sum_{j \in c} e_{i j}$ and $e_{g j}=\frac{1}{|g|} \sum_{i \in g} e_{i j}$ are the mean row and column expression values for $(g, c)$, and $e_{g c}=\frac{1}{|g| \times|c|} \sum_{i \in g, j \in c} e_{i j}$ is the mean expression value over all cells contained in the bicluster $(g, c)$. The threshold $\delta$, a user-defined quantity, represents the maximum allowable dissimilarity within the bicluster. A good bicluster is one for which $\mathcal{G}(g, c)<\delta$ for some $\delta \geq 0$.

Multi-objective evolutionary algorithm, a global search heuristic, has been used for biclustering 12. The maximal set of genes and conditions were generated keeping the "homogeneity" criteria of the biclusters intact. Since these two characteristics of biclusters are conflicting to each other, multi-objective optimization may be employed to model them. To optimize this conflicting pair, the fitness function $f_{1}$ is always maximized while function $f_{2}$ is maximized as long as the residue is below the threshold $\delta$. The formulation is as follows:

$$
\begin{gathered}
f_{1}=\frac{g \times c}{|G| \times|C|}, \\
f_{2}= \begin{cases}\frac{\mathcal{G}(g, c)}{\delta} \text { if } & \mathcal{G}(g, c) \leq \delta \\
0 & \text { otherwise, }\end{cases}
\end{gathered}
$$


where $g$ and $c$ represent, respectively, the number of ones in the genes and conditions within the bicluster, $\mathcal{G}(g, c), \delta$ are as defined earlier, and $G$ and $C$ are the total number of genes and conditions of the initial gene expression array. The Multi-objective GA (NSGA II), in association with the local search procedure discussed in 12 , were used for the generation of the set of biclusters. The algorithm followed is discussed in details in 12 .

\subsection{Correlation Between Gene Pairs}

In this paper we propose a rank correlation-based approach for the extraction of gene interaction networks. A small number of genes participate in a cellular process of interest, being expressed over few conditions. Co-regulated genes are often found to have similar patterns in their gene expression profiles locally, rather than globally. The genes share similar sub-profiles, over a few time points, instead of the complete gene expression profiles. Thus, considering the global correlation amongst genes, i.e., computation of correlation amongst genes employing the complete gene expression data matrix, would not reveal proper relationship between two of them. The Spearman rank correlation provides such a local similarity measure between the two time-series curves, since it is shapebased. The expression profile $e$ of a gene may be represented over a series of $n$ time points. Since the genes in a bicluster are co-expressed, the concept of correlation have been used to quantify their similarity. Instead of the commonly used similarity measures like the Euclidean distance or the Pearson correlation the Spearman rank correlation (RC) have been employed due to its robustness towards outliers and measurement errors [13. Moreover, RC does not assume a Gaussian distribution of points. $R C\left(e_{1}, e_{2}\right)$ between gene expression profile pair $e_{1}$ and $e_{2}$ provides a shape-based similarity measure between the two time-series curves, sampled at $e_{1 i}$ and $e_{2 i}$ over $n$ time intervals. This is expressed as

$$
R C\left(e_{1}, e_{2}\right)=1-\frac{6}{n\left(n^{2}-1\right)} \sum\left[r_{e_{1}}\left(e_{1 i}\right)-r_{e_{2}}\left(e_{2 i}\right)\right]^{2},
$$

where $r_{e_{1}}\left(e_{1 i}\right)$ is the rank of $e_{1 i}$. Here an extended version of the $\mathrm{RC}$ has been used which takes into account the resolving of ties, i.e. $e_{1 j}=e_{1 i}$ for $i \neq j$. The $\mathrm{RC}$ satisfies $-1 \leq R C\left(e_{1}, e_{2}\right) \leq 1$ for all $e_{1}, e_{2}$.

The first preprocessing step is to filter correlation coefficients which contribute minimally towards regulation. This is because often an exhaustive search of the possible interactions between genes is intractable. Next those coefficients are selected whose absolute values are above a detection threshold, suggesting greater correlation amongst the gene pairs. In this way we focus on a few highly connected genes, that possibly link the remaining sparsely connected genes. The correlation range $\left[R C_{\max }, R C_{\min }\right]$ is divided into three partitions each, using quantiles or partition value@1 [14] so that the influence of extreme values or noisy patterns are lessened. It has to be noted that negative correlation between

${ }^{1}$ Quantiles or partition values are the values of a variate which divide the total frequency into a number of equal parts. 
two gene profiles is essentially not zero correlation between them. Furthermore, any correlation coefficient (i) having value above $Q_{2}^{+}$(below $Q_{2}^{-}$) indicates high positive (negative) correlation, and (ii) having value in $\left[Q_{1}^{+}, Q_{2}^{+}\right)\left(\left[Q_{2}^{-}, Q_{1}^{-}\right)\right)$ indicates moderate positive (negative) correlation.

An adjacency matrix is computed as

$$
A(i, j)= \begin{cases}-1 \text { if } & R C \leq Q_{2}^{-} \\ +1 \text { if } & R C \geq Q_{2}^{+} \\ 0 & \text { otherwise }\end{cases}
$$

where we assume absence of self correlations among the genes. Thereafter, a network connecting the various genes is generated.

\section{$3 \quad$ Experimental Results}

\subsection{Network Extraction}

Yeast cell-cycle CDC28 data [15] is a collection of 6178 genes (attributes) for 17 conditions (time points), taken at 10-minute time intervals covering nearly two cycles. The missing values present in the data set are imputed according to the methodology provided in [16] 2 . At first pairwise rank correlation coefficients between gene pairs are computed by eqn. (5) to generate the network architecture from the extracted biclusters. Quantile partitioning is employed next, to choose the strong positive as well as negative correlation links. In this way, the top $\frac{1}{3}$ of the positive and negative links are chosen to be connected in a network. A sample network consisting of three biclusters of sizes 7, 10, and 14, respectively, are shown in Fig. 1] A transcription factor is connected to its target gene by an arrow when such a $T F$-Target pair is found to exist within any of the biclusters. Gene pairs connected by solid lines depict positive correlation, while those connected by dashed lines are negatively correlated. TFs external to the network, but having targets within the network, are connected to their corresponding targets by dotted arrows. As an example, the TF Y H R084W (encircled with solid lines) is a member of the network of 10 genes and has targets in all the three networks. An external TF Y JL056C (encircled with dotted lines) has targets in networks of 7 and 10 genes. The biclusters were biologically validated from gene ontology study, based on the statistically significant GO annotation databas 3 .

Fig. 2 illustrates the expression profiles of the six external TFs GCR1/ $Y P L 075 W, R P N 4 / Y D L 020 C, Y A P 1 / Y M L 007 W, S I N 3 / Y O L 004 W, Z A P 1 /$ $Y J L 056 C$ and $C Y C 8 / Y B R 112 C$ along with the target gene $S S C 1 / Y J R 045 C$, corresponding to the 7 -node bicluster network (generated in terms of pairwise rank correlation values) in Fig. 1] The symbols viz. •, ॰, $\triangle, \square, \diamond, \nabla$, connected by the solid lines, are the expression profiles for the $T F \mathrm{~s}$, respectively, while the dashed line connecting the symbol $\star$ is that corresponding to the regulated gene.

\footnotetext{
${ }^{2}$ LSimpute: accurate estimation of missing values in microarray data with least squares methods.

${ }^{3}$ http://db.yeastgenome.org/cgi-bin/GO/goTermFinder
} 


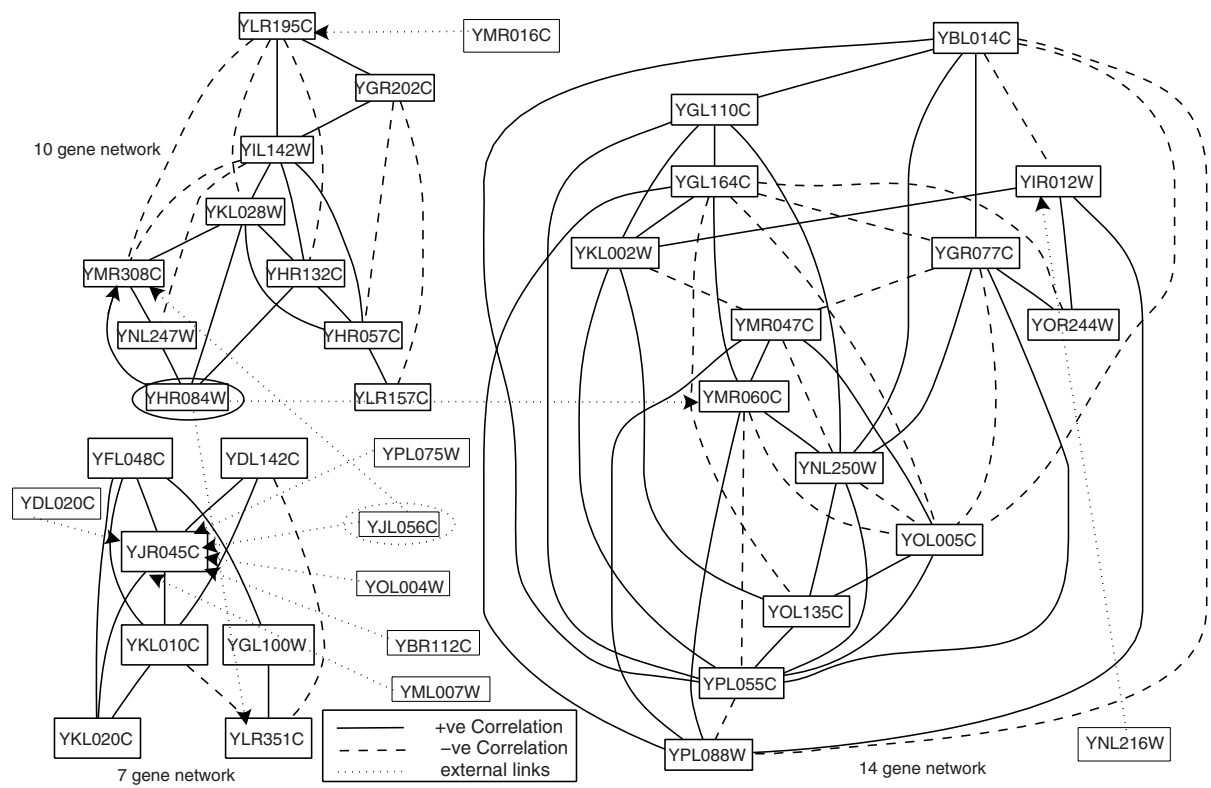

Fig. 1. Network (bicluster) of 10 genes connected by transcription factor $Y H R 084 W$ to networks (biclusters) of 7 and 14 genes

The behavior of $G C R 1 / Y P L 075 W$ with the target is considered as an example. Here the two genes are found to behave almost simultaneously over conditions 0-40 minutes, while the same two demonstrate a marked inverted relationship during conditions 40-80 minutes. Again the time span of 110-130 minutes displays a shifted response of the target as compared to the $T F$. However, the expression profiles of $C Y C 8 / Y B R 112 C$ and the target appear to share a strong simultaneous relationship almost along the entire time span. So the genes are found to behave similarly in their gene expression sub-profiles.

\subsection{Biological Validation}

During the prediction of regulatory networks [17 the genes $Y H R 084 W$ $Y L R 351 C$ were reported to form a TF-Target pair. We also obtained the summary of the TF-Target pair Y H R084W-YLR351C (Fig.10 in terms of Molecular Function, Biological Process and Cellular Component from the Saccharomyces Genome Database (SGD) 4 . From our calculations we have also confirmed that an interaction exists between the target and its $T F$. It is reported in the database that the biological process involving protein $Y L R 351 C$ is not fully understood as yet and $Y H R 084 W$ has transcription factor activity. It becomes more difficult when one attempts to extract some biologically meaningful information

\footnotetext{
${ }^{4}$ A scientific database of the molecular biology and genetics of the yeast Saccharomyces cerevisiae - http://db.yeastgenome.org/
} 


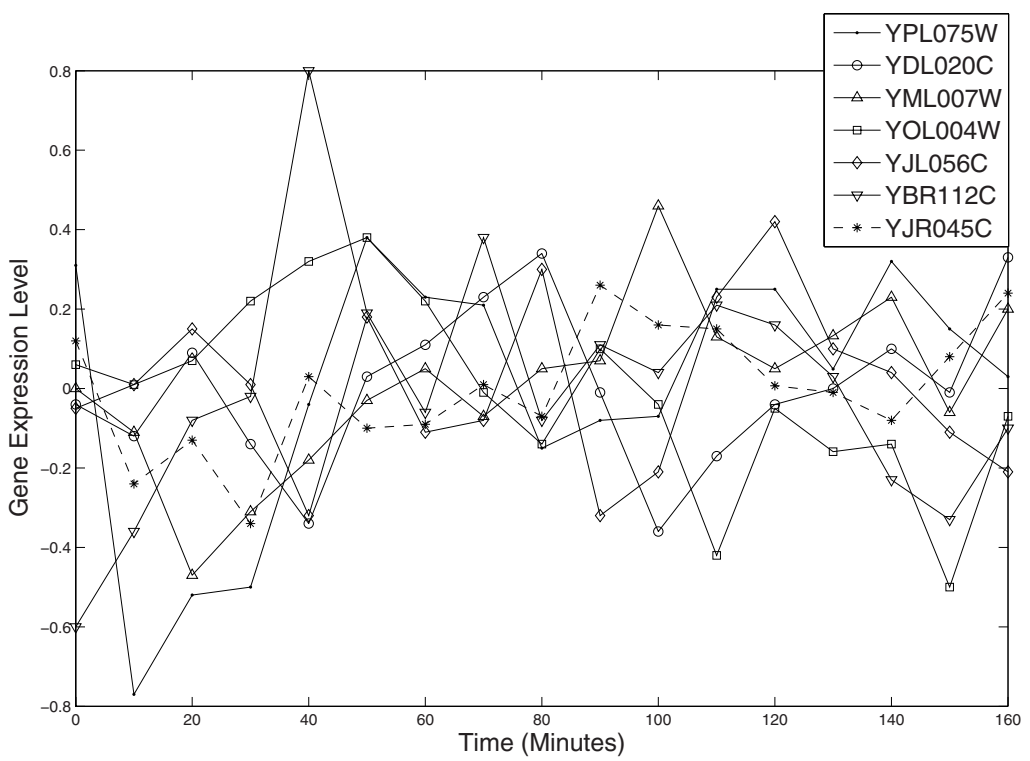

Fig. 2. Expression profile of six transcription factors and target YJR045C, in the 7node network

involving these two entities. From such scanty information our method has been able to identify that there exists a link between a $T F$ and its target. From their cellular components we model, as an efficacy of the biclustering, the transcription of $Y L R 351 C$ by $Y H R 084 W$ occurring inside the nucleus, and then the regular translation mechanism follows. In like manner for the $T F$-Target pair of $Y P L 075 W$ and $Y J R 045 C$ (Fig. (1) reported in 17), we obtained their summary from SGD and found $Y P L 075 \mathrm{~W}$ to be transcriptional activator of genes involved in glycolysis while $Y J R 045 C$ has ATPase, enzyme regulator and protein transporter activity. Again we were able to predict that $Y P L 075 \mathrm{~W}$ is involved in the transcription of $Y J R 045 C$ and would go into the glycolysis process.

One can arrive at similar kind of conclusions, for the rest $T F$-Target pairs, with a certain definite degree of confidence. As relevant literature in this area are really very sparse a large number negative results is only expected. Our algorithm has not yet detected any false positive or false negative $T F$-Target pairs, which is consistent with the information available either in the literature or in the databases.

\section{Conclusions and Discussion}

In this paper we have introduced an approach based on multiobjective evolutionary biclustering and subsequent extraction of rank correlated gene pairs for the extraction of gene interaction networks. Biologically relevant small biclusters 
were obtained, using time-series gene expression data from Yeast. These were validated using the statistically significant GO annotation database. The pairwise rank correlation coefficients among gene pairs were computed by eqn. (5) followed by the quantile partitioning to select the strong positive as well as negative correlation links. The strongly correlated genes were then chosen to be connected in a network. TF-Target gene pairs in the network, shown in Fig. 1] were found to exhibit strong correlations. We tried to model the interaction among them from information available in the literature/databases viz., SGD. We have also analyzed the expression profiles of the regulator and the regulated genes which reveals several complex (time shifted, inverted, simultaneous, etc.) relationships between them. The sparse nature of gene regulatory networks was reflected well on choosing Spearman rank correlation as the similarity measure.

\section{References}

1. Mitra, S., Pedrycz, W.: Special Issue on Bioinformatics. Pattern Recognition 39 (2006)

2. Eisen, M.B., Spellman, P.T., Brown, P.O., Botstein, D.: Cluster analysis and display of genome-wide expression patterns. Proceedings of National Academy of Sciences USA 95, 14863-14868 (1998)

3. Tavazoie, S., Hughes, J.D., Campbell, M.J., Cho, R.J., Church, G.M.: Systematic determination of genetic network architecture. Nature Genetics 22, 281-285 (1999)

4. Gasch, A.P., Eisen, M.B.: Exploring the conditional coregulation of yeast gene expression through fuzzy $k$-means clustering. Genome Biology 3, research0059.1$0059.22(2002)$

5. Ji, L., Tan, K.L.: Identifying time-lagged gene clusters using gene expression data. Bioinformatics 21, 509-516 (2005)

6. Madeira, S.C., Oliveira, A.L.: A Linear Time Biclustering Algorithm for Time Series Gene Expression Data. In: Casadio, R., Myers, G. (eds.) WABI 2005. LNCS (LNBI), vol. 3692, pp. 39-52. Springer, Heidelberg (2005)

7. Cheng, Y., Church, G.M.: Biclustering of gene expression data. In: Proceedings of ISMB 2000, pp. 93-103 (2000)

8. Bansal, M., Belcastro, V., Ambesi-Impiombato, A., di Bernardo, D.: How to infer gene networks from expression profiles. Molecular Systems Biology 3, 1-10 (2007)

9. Jong, H.D.: Modeling and simulation of genetic regulatory systems: A literature review. Journal of Computational Biology 9, 67-103 (2002)

10. Zhang, Y., Zha, H., Chu, C.H.: A time-series biclustering algorithm for revealing co-rregulated genes. In: Proceedings of the International Conference on Information Technology: Coding and Computing (ITCC 2005), pp. 1-6 (2005)

11. Madeira, S.C., Oliveira, A.L.: Biclustering Algorithms for Biological Data Analysis: A Survey. IEEE Transactions on Computational Biology and Bioinformatics 1, 24 $45(2004)$

12. Mitra, S., Banka, H.: Multi-objective evolutionary biclustering of gene expression data. Pattern Recognition 39, 2464-2477 (2006)

13. Balasubramaniyan, R., Hllermeier, E., Weskamp, N., Kamper, J.: Clustering of gene expression data using a local shape-based similarity measure. Bioinformatics 21, 1069-1077 (2005)

14. Davies, G.R., Yoder, D.: Business Statistics. John Wiley \& Sons, London (1937) 
15. Cho, R.J., Campbell, M.J., Winzeler, L.A., Steinmetz, L., Conway, A., Wodicka, L., Wolfsberg, T.G., Gabrielian, A.E., Landsman, D., Lockhart, D.J., Davis, R.W.: A genome-wide transcriptional analysis of the mitotic cell cycle. Molecular Cell 2, 65-73 (1998)

16. Bo, T.H., Dysvik, B., Jonassen, I.: Lsimpute: accurate estimation of missing values in microarray data with least squares methods. Nucleic Acids Research 32, 1-8 (2004)

17. Qian, J., Lin, J., Luscombe, N.M., Yu, H., Gerstein, M.: Prediction of regulatory networks: genome-wide identification of transcription factor targets from gene expression data. Bioinformatics 19, 1917-1926 (2003) 